\title{
A more superior preformed particle gel with potential application for conformance control in mature oilfields
}

\author{
Paul Tongwa $\cdot$ Bai Baojun
}

Received: 14 March 2014/Accepted: 10 August 2014/Published online: 7 September 2014

(C) The Author(s) 2014. This article is published with open access at Springerlink.com

\begin{abstract}
The present work presents a nanocomposite preformed particle gel for conformance control in mature oilfields. This product was observed to have a better performance than conventional hydrogels without nanomaterials. Preformed particle hydrogel was designed by the reaction of monomer, initiator, crosslinker, additives, and calcium montmorillonite nanomaterial, whereas conventional hydrogel is designed from just monomer, initiator, crosslinker and additives. The presence of nanomaterial in hydrogel design affords it tremendous improvement in nanocomposite gel properties and behavior compared to conventional hydrogels without any nanomaterial. Additionally, swelling performance, post-degraded gel viscosity, and long-term thermal resistance of nanocomposite gel increased by several orders of magnitude compared to hydrogels with no nanomaterial. Environmental Scanning Electron Microscopy (ESEM) revealed the presence of a very dense 3-D network compared to hydrogels with no nanomaterial. On incorporation of nanomaterial, increase in gel strength of up to $2,150 \%$ was observed. Thus, they are potential replacements for existing preformed particle gels in mitigating permeability variations in mature reservoirs.
\end{abstract}

Keywords Enhanced oil recovery - Conformance control · Nanocomposite gels · Polymer flooding · Permeability variations $\cdot$ Mature oilfields

P. Tongwa $\cdot$ B. Baojun $(\bowtie)$

Department of Petroleum Engineering, Missouri University of Science and Technology, 1870 Miner Circle, Rolla, MO 65409, USA

e-mail: baib@mst.edu

\section{Introduction and background}

The fact that the oil and gas industry is spending a lot of money and using every available cutting-edge technology to find oil in very risky and unfavorable terrains such as the deep seas and polar regions of the earth is a pointer to the fact that primary and secondary oil production from existing fields is reaching peak production. However, these existing fields still contain significant and unrecoverable quantities of hydrocarbons which cannot be recovered by current available technologies. Rather than explore for oil in such risky terrains, why not optimize oil production from already existing, mature fields which have a well-known production history and performance? Such enormous and untapped amount of hydrocarbons in already existing, mature, left-behind fields is the goal of Enhanced Oil Recovery (EOR, also called tertiary production). EOR methods are crucial to a continuous world supply of oil.

As reservoirs mature, oil production declines while water production rises. Excess and unwanted water production from these mature fields is one problem that has plagued the oil industry for decades. Excess water production is a frequent problem that occurs in mature reservoirs as a result of long-term water flooding. Such excess water production usually results in increased environmental concerns, increased levels of corrosion and scale and ultimately leads to early shut-in of wells that still contain significant volumes of hydrocarbons (Liu et al. 2006; Bai et al. 2007a).

One fundamental reason for a decline in oil production with time is the existence of fractures and permeability variations that exist between the different layers of the reservoir. Fractures present a water-thief zone through which injected flood water channels through, from the injector to the producer, thereby leaving hydrocarbons in the low permeability (non-fractured) zones untouched. The

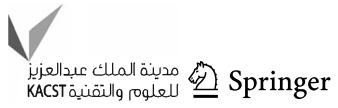


injected flood water follows the path of least resistance (high permeability zones), bypassing large amount of oil in low permeability matrix. This leads to increased, unwanted water production and poor oil recovery. Therefore, plugging reservoir fractures and thus correcting reservoir heterogeneity (that is conformance control) are key to an increased oil production, and hence the reason for this work.

In an attempt to mitigate excess water production and hence increase hydrocarbon production, hydrogels are often injected near wellbore or far-wellbore to preferentially seal fractures or higher permeability zones (Tongwa et al. (2013a, 2013b); Bai et al. (1999, 2007b, 2008); Liu et al. 1999). Hydrogels as fracture-plugging and fluid diverting materials have been employed in conformance control (profile modification) and in the control of excess water production during EOR applications [Bai et al. (1999, 2007a, b, 2008); Zhang and Bai 2011; Vossoughi (2000); Wang et al. (2001, 2003)]. Mechanistically, hydrogel is injected to seal high permeability and fracture zones, such that when injected fluids are injected, they will be forced or redirected to the low permeability, unswept oil-rich zones, sweeping out oil from them, leading to additional oil production. This, in summary, is the goal of any gel treatment work. This process is called profile modification or permeability modification. As the term implies, the process seeks to even out or correct the sharp difference in permeabilities that exist in the different formation layers, creating a homogeneous reservoir.

Over the years, different types of gel treatments have been utilized in an attempt to solve conformance control problems. Initially, industry started using in situ gels in which gelling solution is injected into reservoir and crosslinking of gelling solution to form 3-D bulk gel occurs downhole. This technology was quickly dropped due to its inherent disadvantages such as selective injectivity, possible damage to low permeability zones, dispersion and dilution of gelant, syneresis, dehydration, and inadequate control of gelation time (Seright 1990; Young et al. 1988; Asghari 1999; Bryant et al. 1996; Willhite et al. 1986).

In an attempt to overcome the various limitations of in situ gel technology, industry experts and researchers developed a novel technology to address conformance control problems called preformed gel technology. Preformed gels are three-dimensional, hydrophilic crosslinked polymers which, in contact with water, swell but do not dissolve as a result of a chemical or physical crosslinking and often than not will undergo a volume phase change when surrounding conditions such as temperature, salinity or pH change (Wen-Fu and Sung-Chuan 2006; Kytai and Jennifer 2002). The novelty and main difference between this technology and in situ gel technology is that with preformed gel, gel formation takes place at the surface well ahead before injection, whereas with in situ gelation,

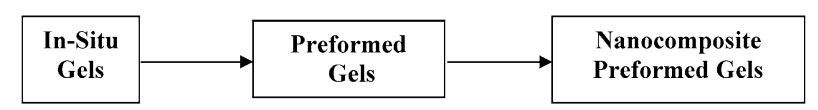

Prior technology $\longrightarrow$ Current technology $\longrightarrow$ Future technology

(Seright, 1988) (Bai, 2001)

Fig. 1 Progress in gel development for conformance control

crosslinking and gel formation occur downhole in the reservoir (Bai et al. 2013; Frampton et al. 2004). This technology was revolutionary in that it addressed some of the problems posed by in situ gelation such as dilution and dispersion of gelant, chromatographic separation of gelant solution, dehydration, syneresis, and most importantly damage of low permeability zones.

However, despite the tremendous advantages of preformed gels over in situ gels, preformed gels have not as yet provided an all-encompassing solution to the problem of conformance control and reservoir heterogeneity. Some of the limitations of preformed gels include: (a) Mechanical: inadequate strength and toughness, (b) thermal: inadequate thermal resistance to withstand very extreme reservoir conditions, shorter degradation time, (c) swelling: inadequate swelling ability, (d) elasticity: inadequate gel elasticity.

Thus, there is a present need to provide a technology that surpasses the performance of current preformed gel technology. The current work is an extension of existing preformed gel technology by the incorporation of nanomaterials in gel design for improved mobility control (Fig. 1).

Conventional preformed gel synthesis involves a multicomponent reaction that incorporates monomer, initiator, and crosslinker. However, our nanocomposite preformed particle gel (nanocomposite PPG) synthesis involves a multi-component reaction that incorporates monomer, initiator, crosslinker, and nanomaterial (Fig. 2).

\section{Materials and methods}

\section{Materials}

Monomer used in this study, acrylamide, AM (98.5\%), was purchased by Alfa Aesar company (Ward Hill, MA) as a white granular solid and is completely water soluble. It was used as received. Crosslinker used is Polyethylene glycol diacrylate (PEG-200) purchased from Sigma Aldrich company. Nanoclay used in this study, calcium montmorillonite was received with courtesy from Southern Clay Products (SCP) Inc. Potassium persulfate $\left(\mathrm{K}_{2} \mathrm{~S}_{2} \mathrm{O}\right.$, $\mathrm{KPS}$ ) as redox initiator was employed to polymerize the monomer solutions of acrylamide and was purchased from Sigma Aldrich Company. Sodium Chloride, $\mathrm{NaCl}(99.8 \%)$ 
Fig. 2 Synthesis of existing and proposed preformed gel technology

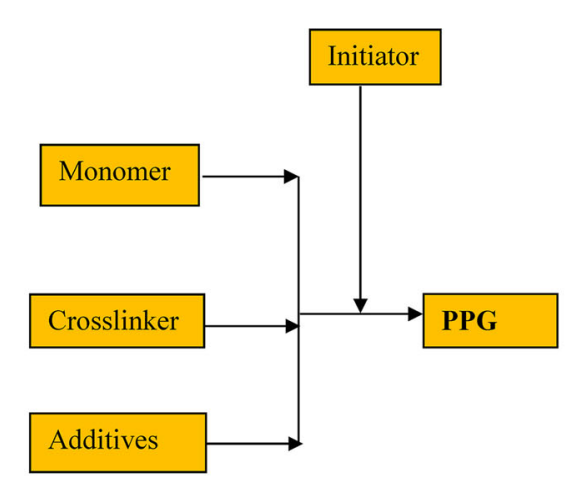

(a) Existing preformed gel technology

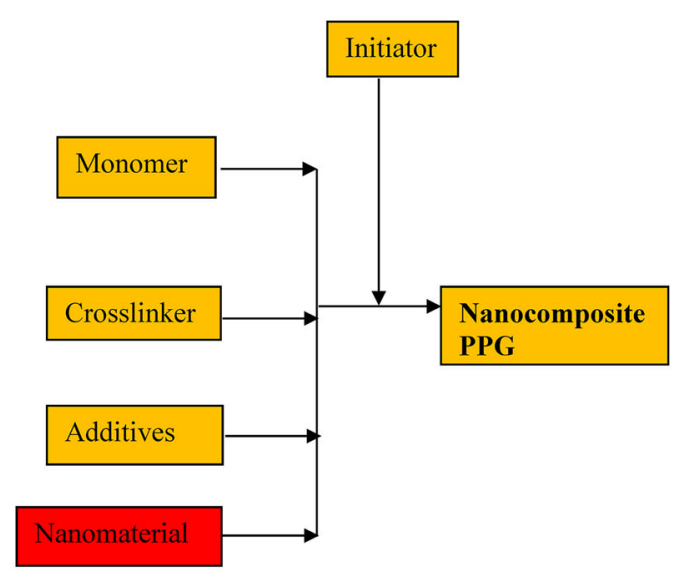

(b) Proposed nanocomposite gel technology was purchased from Fisher Scientific Inc. and used as received. Distilled water was used for the synthesis while $1 \%$ brine solution and formation water were used for swelling experiments.

The following is one example to illustrate the synthesis process for nanocomposite gels. First, $30 \mathrm{~g}$ of AM was dissolved in $100 \mathrm{~g}$ of distilled water in a doublenecked flat-bottomed reactor equipped with inlet and outlet tubes for nitrogen gas. The mixture was stirred at room temperature for $10 \mathrm{~min}$. Then, $0.2 \%(0.26 \mathrm{~g})$ of Calcium Montmorillonite was added to the solution and stirred vigorously for about $24 \mathrm{~h}$ to ensure complete exfoliation of clay nanomaterial. Then, $250 \mathrm{ppm}$ of the labile crosslinker PEG-200 was added to the mixture and stirred for $10 \mathrm{~min}$. The mixed solution was then purged with nitrogen gas for $30 \mathrm{~min}$ before $100 \mathrm{ppm}$ of KPS initiator was added to the solution. This resulting solution was kept for $10 \mathrm{~h}$ at $40{ }^{\circ} \mathrm{C}$ in a water bath to ensure complete gelation. The strong and elastic bulk gel formed was cut into small pieces. It was then purified by soaking in a large amount of distilled water for 3 days to remove the unreacted monomers and additives, followed by drying in an oven at $60{ }^{\circ} \mathrm{C}$ until the weight could not change any more. The dried gel solids were crushed into very small particle sizes, called preformed particle gels (PPGs), by blending in a blender machine (Black \& Decker). PPGs with the particle size between 80 and 100 mesh $(180-250 \mu \mathrm{m})$ were selected through the standard testing sieves (Fisher Scientific Company) for further characterization and evaluation. For gels with no nanomaterial, the same procedure was followed except that nanoclay was excluded.

\section{Methods}

The following properties were evaluated for the nanocomposite gels:

\section{Swelling kinetics}

The essence of swelling measurements is to ascertain the maximum swelling capacity of gels to determine its ability to swell and plug reservoir fractures and high permeability matrices. A $1 \%$ gel solution was prepared by immersing $0.25 \mathrm{~g}$ of the dry particle gel in $24.75 \mathrm{~g}$ of $1 \% \mathrm{NaCl}$ brine solution and formation water, respectively. The Swelling ratio of the gels was calculated from the following equation:

Swelling ratio $=\mathrm{V}_{\mathrm{s}} / \mathrm{V}_{\mathrm{i}}$

where, $V_{i}$ is the volume of dry gel and,

$\mathrm{V}_{\mathrm{s}}$ is the volume of swollen gel

\section{Gel strength (rheology) test}

The rheological properties of hydrogels were measured using a Haake RheoScope RO1 version 3.61.0000 from Thermo Scientific. The sensor used for all measurements was PP20 with a gap of $2 \mathrm{~mm}$. The samples were cut in uniform dimensions with diameter of $2 \mathrm{~cm}$ and height of $2 \mathrm{~mm}$. The measurements were set as an oscillation model and frequency experiments were first performed in the range of $1-15 \mathrm{~Hz}$ to establish the extent of the linear viscoelastic region. Based on the data, all subsequent oscillation time-dependent experiments were performed at a fixed frequency of $1 \mathrm{~Hz}$ and controlled stress (CS) of 1.0 Pa to obtain the values of elastic modulus, (G') and viscous modulus, $\left(G^{\prime \prime}\right)$ as a function of time. All runs were repeated at least three times.

\section{Thermostability tests}

Long-term thermal stability testing were carried out in the key apparatus shown in Fig. 3. First, $0.12 \mathrm{~g}$ of dry particle gels was measured into each ampoule, and then $11.88 \mathrm{~g}$ of 
Fig. 3 Manifold used to seal ampoule

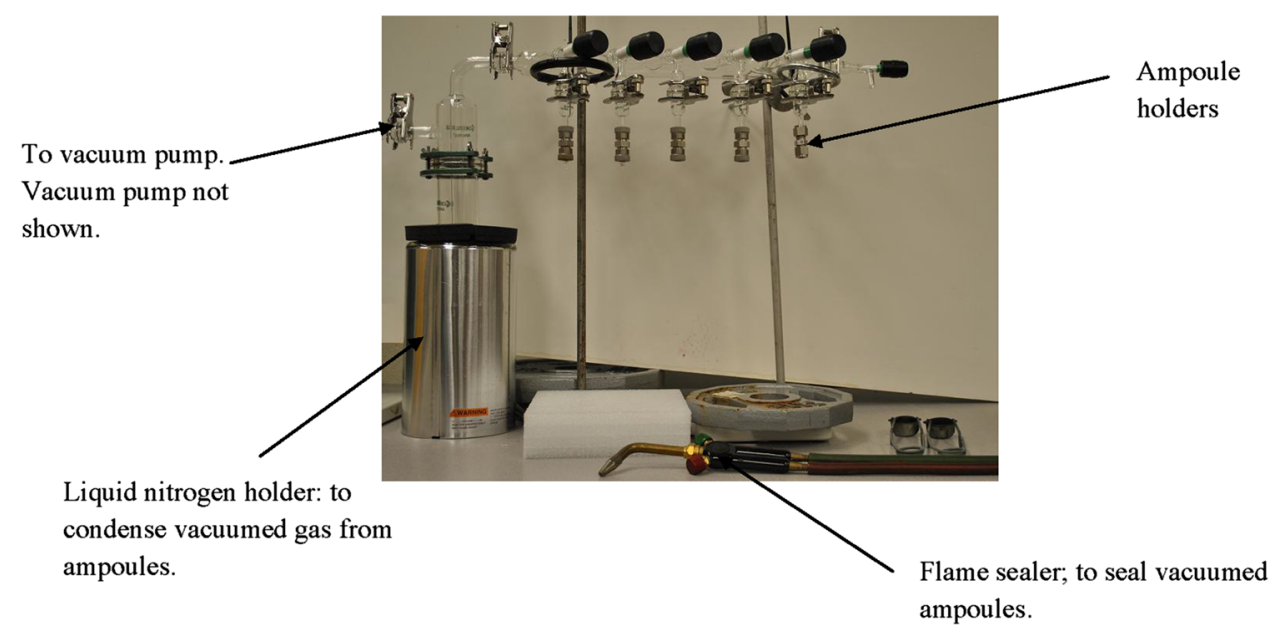

Fig. 4 An obvious improvement in hydrogel between gels with nanomaterials and those without nanomaterials. Gel strength is seen to increase with increasing calcium nanomaterial concentration mechanical strength is observed

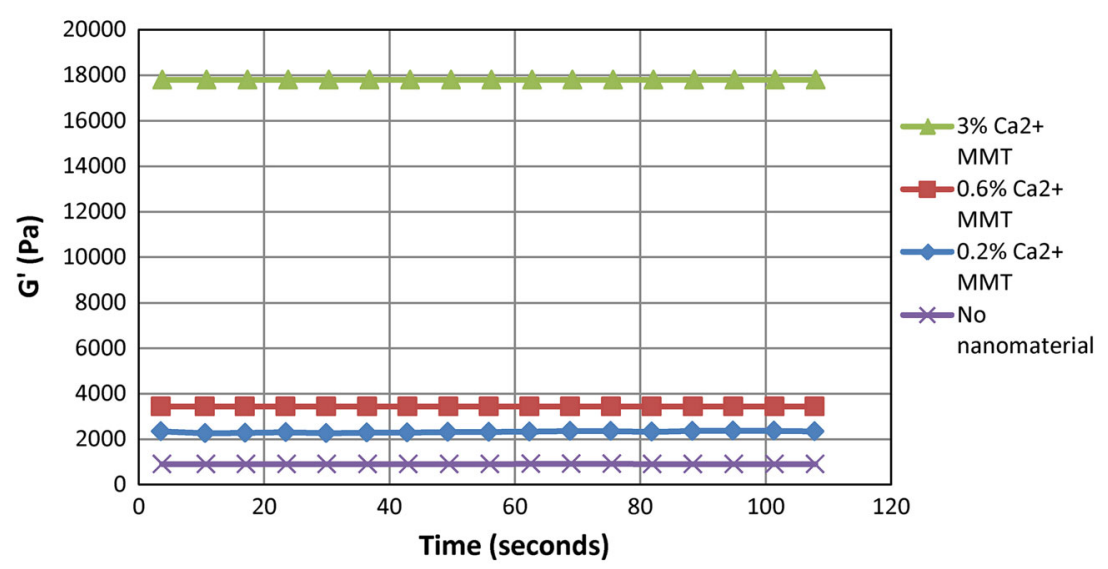

brine $(1.0$ wt $\% \mathrm{NaCl})$ solution was injected into each ampoule. Therefore, the gel concentration was set at $10,000 \mathrm{ppm}$ and $12 \mathrm{~g}$ total for each ampoule. The ampoules were placed one at a time into the manifold. The valves were closed, and a vacuum pump was started. After the ampoules had been attached to the manifold, each valve was slowly opened one at a time to minimize any rush of liquid or gas out of the ampoule into the manifold. The vacuum pump continued to run at $-25 \mathrm{psi}$ for about half an hour to remove the dissolved gases in the liquid sample, including any trace of dissolved oxygen that might have remained in the sample. Next, the ampoules were flame sealed in place. The sealed ampoules were weighed using an analytical balance with an accuracy of $0.0001 \mathrm{~g}$, and then placed in an oven and aged at 45,60 and $80^{\circ} \mathrm{C}$. After the specified aging times, one ampoule was taken out of the oven and cooled to room temperature. This ampoule was reweighed to confirm that none of the solution had leaked. A loss of about $0.001 \mathrm{~g}$ or more indicated possible leakage from this ampoule. In that case, another ampoule would be used for a post-aging measurement.

\section{Environmental SEM evaluation}

Environmental scanning electron microscopy (ESEM) studies were employed to study the porous network structure of the gel. This gives information about pore-interconnectivity and swelling propensity. After the particle gels were completely swollen in brine, ESEM was used to examine the surface morphology of the swollen particle gel. Swollen nano-PPG samples were mounted on metal stubs at a low vacuum degree (4.6 Torr), and a relatively low temperature (near $0^{\circ} \mathrm{C}$ ) was observed. The samples first underwent a freeze process in the chamber of an FEI Quanta 600 FEG extended vacuum scanning electron microscope. To emphasize the gel microstructure, the following ESEM imaging protocol was followed: the temperature and pressure were decreased simultaneously from $0{ }^{\circ} \mathrm{C}$ and 4.6 Torr to $-5^{\circ} \mathrm{C}$ and $2-3$ Torr, thereby freezing the sample; the temperature was then allowed to rise to $20{ }^{\circ} \mathrm{C}$ with a rate of $2{ }^{\circ} \mathrm{C} /$ minute at $2-3$ Torr pressure to sublimate water from the sample at a relative humidity of $12.5 \%$. 
(a)



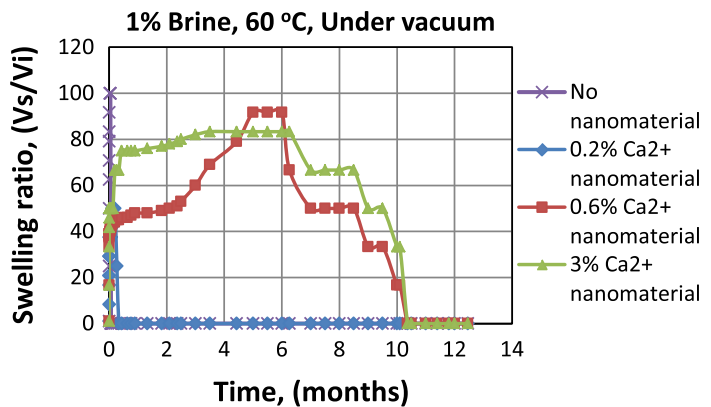

Time, (months)

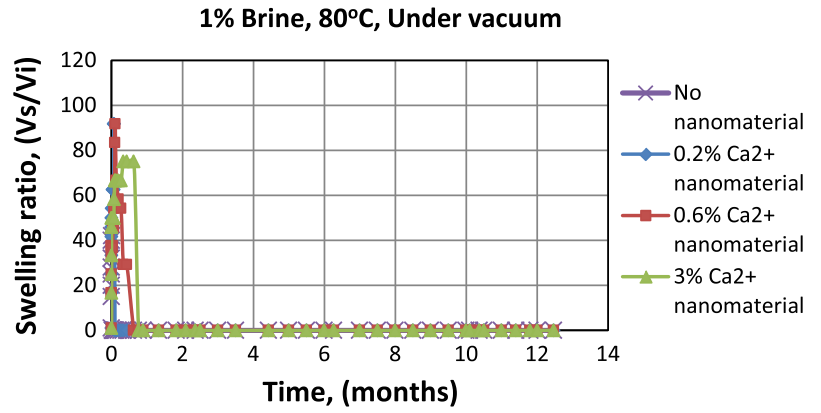

(b)

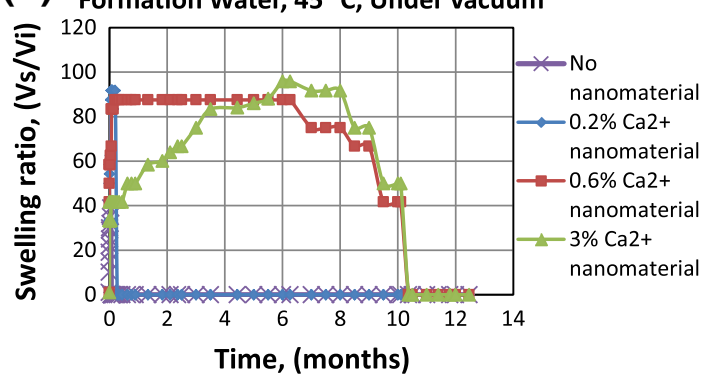

Formation Water, $60^{\circ} \mathrm{C}$, Under vacuum

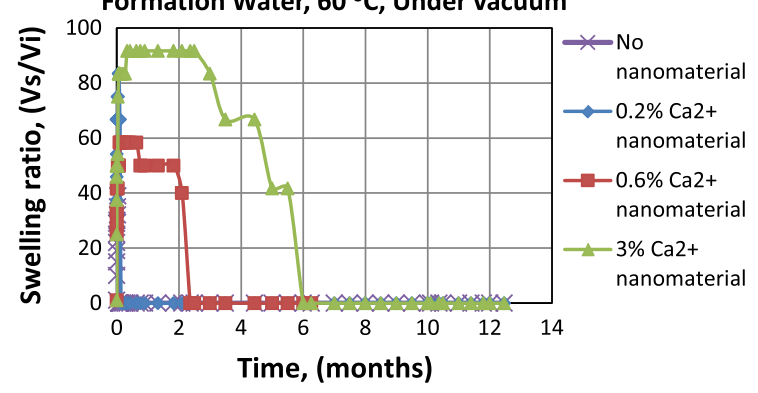



Fig. 5 Long-term thermal stability of $\mathrm{Ca}^{2+}$ nanocomposite PPG under anaerobic conditions and In a $1 \%$ brine solution and b Formation water. $\mathrm{Ca}^{2+}$ nanocomposite PPG took a significantly longer time to degrade in anaerobic environment than in aerobic environment. This is because oxygen causes premature gel degradation. In the absence of oxygen, $\mathrm{Ca}^{2+}$ nanocomposite PPG took longer to degrade

\section{Viscosity measurements}

The viscosity of nanocomposite PPG after thermal degradation was measured. After an extended time period, gel degrades into a viscous polymer solution. This viscous polymer solution will then move into deeper regions of the reservoir to increase the viscosity of the flood water and boost polymer flooding process. Thus the higher the postdegradation gel viscosity, the better its ability to improve polymer flooding process. The viscosity of the degraded 

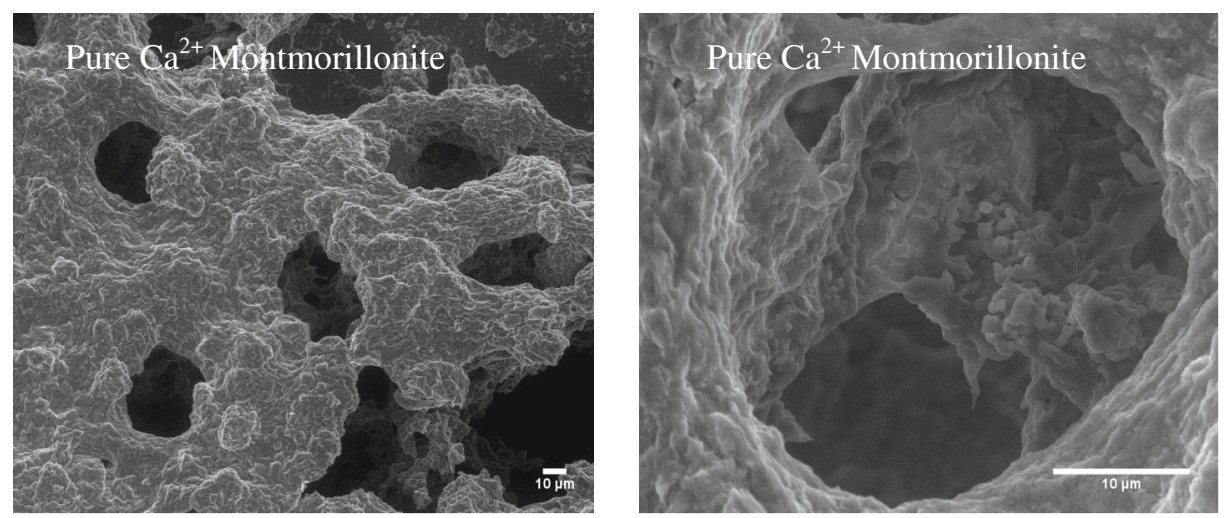

(a) Pure $\mathrm{Ca}^{2+}$ Montmorillonite Nanomaterial

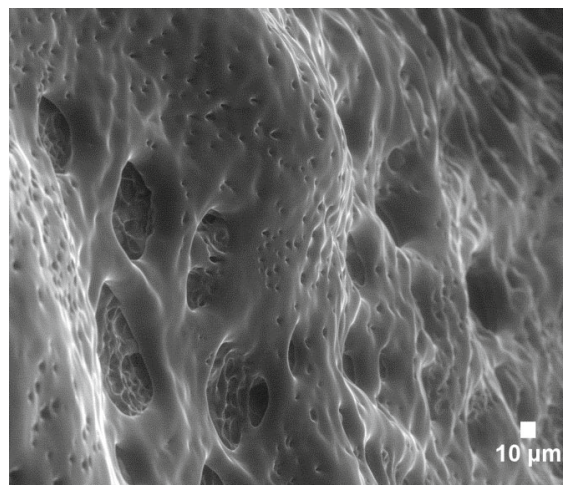

(b) Pure polyacrylamide polymer

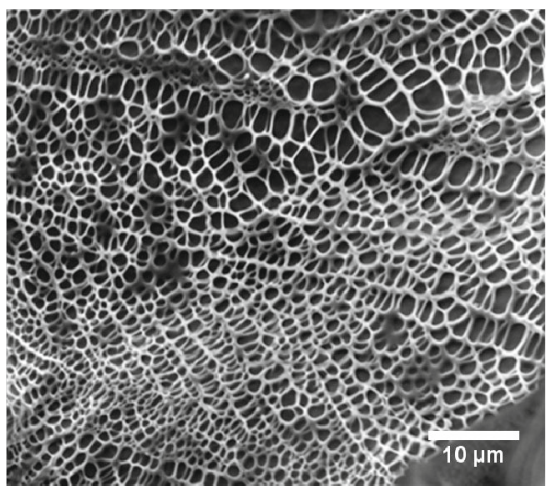

(c) Fine, smooth network structure of hydrogel with no nanomaterial (Jia, 2011)
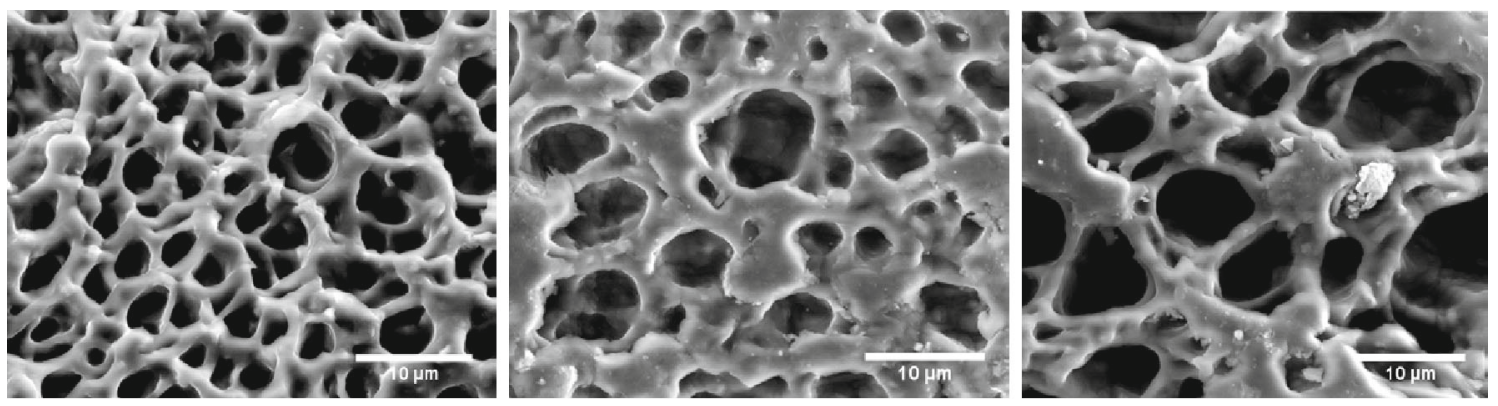

(d) ESEM micrographs of $\mathrm{Ca}^{2+}$ nanocomposite PPG swelled in $1 \%$ brine.
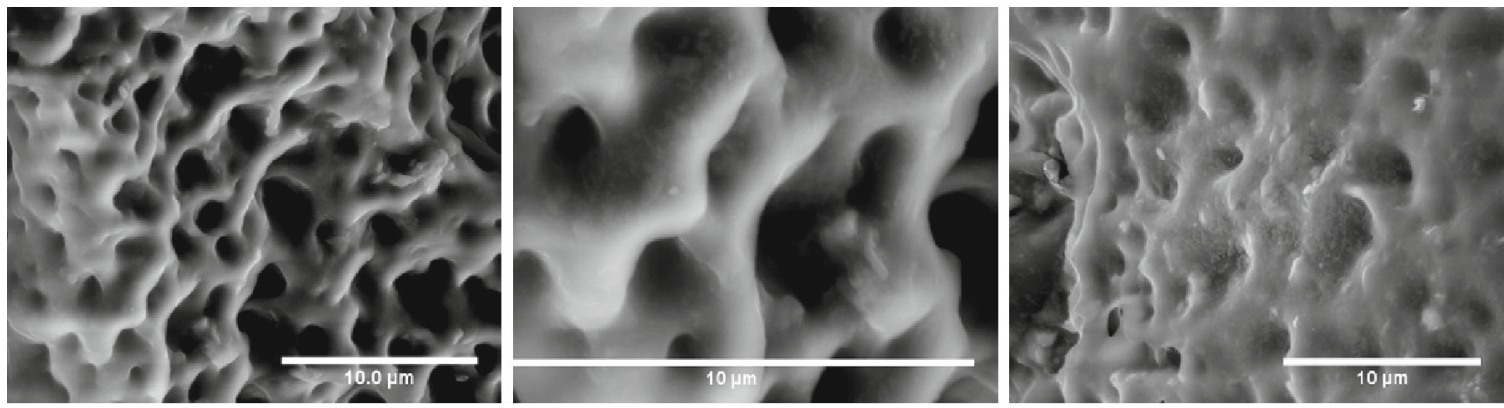

(e) ESEM micrographs of $\mathrm{Ca}^{2+}$ nanocomposite PPG swelled in distilled water. 
4Fig. 6 Before-degradation environmental scanning electron microscopy (ESEM) Micrographs of: a Pure calcium montmorillonite nanomaterial. b Pure polyacrylamide (PAM) polymer. c Hydrogel with no nanomaterial. A fine, smooth network structure is observed. d $\mathrm{Ca}^{2+}$ nanocomposite PPG swelled in $1 \%$ brine as the solvent. A very conspicuous porous network structure is seen. $\mathbf{e ~} \mathrm{Ca}^{2+}$ nanocomposite PPG swelled in distilled water. In distilled water, the conspicuous porous network structure diminishes. We do not fully understand the reason for this occurrence

solution was measured using a Brookfield viscometer with a shear rate of 0 RPM to 100 RPM using a \#18 or \#34 spindle. The viscosity measured at 6 RPM was recorded as the reported value.

\section{Results and discussion}

A detailed vis-à-vis comparison of currently existing and novel nanocomposite hydrogels reveals that the latter far supersedes existing hydrogels in terms of product performance and usefulness. A summary of the results obtained for the different tests carried out are presented below.

Improvement in mechanical performance of nanocomposite hydrogel compared to hydrogel with no nanomaterial

The rheology behavior of $\mathrm{Ca}^{2+}$ Nanocomposite PPGs and PPGs with no nanomaterial was studied. The variation in elastic modulus $\left(G^{\prime}\right)$ with time for $\mathrm{Ca}^{2+}$ Nanocomposite PPG with $0.2,0.6$, and $3 \%$ calcium nanomaterial is presented in Fig. 4 and is compared against hydrogel with no nanomaterial. It is observed from Fig. 4 that the elastic modulus significantly increases with increasing nanomaterial concentration. The elastic modulus of hydrogel with no nanomaterial is at the lowest value of $800 \mathrm{~Pa}$, while hydrogel with $3 \%$ calcium nanomaterial has an elastic modulus of about $18,000 \mathrm{~Pa}$. Clearly, an increase in gel strength is observed as nanomaterial is introduced.

Improvement in thermal resistance compared to hydrogel with no nanomaterial

Figures 5 presents long-term testing in anaerobic conditions using both $1 \%$ brine and formation water. The reason for testing gel degradation under anaerobic conditions was to simulate reservoir environment where oxygen amounts are minimal. Oxidation in presence of oxygen causes gel to degrade much faster. Therefore, it was necessary to remove every trace of oxygen to avoid premature gel breakdown.

Once injected downhole into fractures or high permeability streaks, the long-term thermal resiliency of hydrogels to continuously seal fractures under adverse reservoir conditions is important. Without long-term endurance, gels rapidly degrade, leading to a re-opening of an already sealed fracture, thus re-creating a water-thief channel. Therefore, ensuring hydrogels can adequately seal fractures over a prolonged period of time is paramount. Figure 5 presents long-term thermal stability testing of hydrogels in both $1 \%$ brine and formation water. As is clearly seen from Fig. 5, hydrogels with no nanomaterial rapidly degraded within days whereas for hydrogels with nanomaterial, degradation occurred over a year in some cases and in others hydrogels have not degraded yet. Additionally, we observed that an increase in nanomaterial concentration led to an increase in long-term thermal resistance of hydrogels. This is as expected because increasing nanomaterial concentration leads to an increased participation of nanomaterial in the gelation process, affording a stronger gel. Such dramatic improvement in long-term thermal stability of nanocomposite hydrogels is one key reason why we believe they are potentially valuable in conformance control applications.

Evaluation of $\mathrm{Ca}^{2+}$ nanocomposite PPG microstructure and morphology, before and after degradation

\section{Environmental scanning electron microscopy imaging} of $\mathrm{Ca}^{2+}$ nanocomposite PPG before its degradation

A detailed microscopic study of $\mathrm{Ca}^{2+}$ Nanocomposite PPG was done using an environmental scanning electron microscope (ESEM), and was compared against hydrogels with no nanomaterial. Studying the network structure of hydrogel is important because it gives us information about pore-interconnectivity. This information is useful in understanding the mechanisms of gel swelling behavior, gel strength after it swells, and perhaps even its thermal resistance ability.

Figure 6a presents an ESEM micrograph of pure Calcium Montmorillonite nanomaterial. Figure $6 \mathrm{~b}$ presents an ESEM micrograph of pure polyacrylamide (PAM) polymer. Figure 6c presents a micrograph of hydrogel with no nanomaterial. The micrographs of $\mathrm{Ca}^{2+}$ Nanocomposite PPG swelled in $1 \%$ Brine is presented in Fig. 6d. A very conspicuous porous network structure is seen. Figure 6e presents the ESEM micrographs of $\mathrm{Ca}^{2+}$ nanocomposite PPG swelled in distilled water. In distilled water, the conspicuous porous network structure diminishes.

Contrasting the hydrogel with no nanomaterial versus the hydrogel with nanomaterial, (that is Figs. 6c versus Figs. $6 \mathrm{~d}-\mathrm{e}$ ), we observe that although a porous interconnected network structure is seen in both nanocomposite and non-nanocomposite hydrogels, in $\mathrm{Ca}^{2+}$ Nanocomposite PPG, however (Figs. 6d, e), the network structure is more 
conspicuous, thicker, denser, and corrugated whereas in hydrogels with no nanomaterial the network structure is finer, less dense, and smooth. Obviously, we say that the presence of Calcium Montmorillonite nanomaterial in nanocomposite hydrogel affords this difference.

We also mention that when brine was used as the solvent, the network structure is extremely conspicuous (Fig. 6d) such that the pores are very clearly visible. However, when distilled water was used as the solvent, the conspicuousness of the network structure diminishes. We do not fully understand the reason for this occurrence. An opposite phenomenon was observed by Nelea et al. 2007. In their work, they instead observed that the network structure was very conspicuous when distilled water was used, and when brine was used, the network was less visible.

Environmental scanning electron microscopy imaging of $\mathrm{Ca}^{2+}$ nanocomposite PPG after its degradation

After the $\mathrm{Ca}^{2+}$ nanocomposite PPG degraded, ESEM micrographs were again taken of the degraded sample. Figure $7 \mathrm{a}$ shows the micrograph of the degraded hydrogel with no nanomaterial. Figures $7 \mathrm{~b}$ shows the micrographs of a $3 \%$ degraded $\mathrm{Ca}^{2+}$ nanocomposite PPG. As is clearly seen in both non-nanocomposite (Fig. 7a) and nanocomposite gel (Figs. 7b), the homogenous porous network

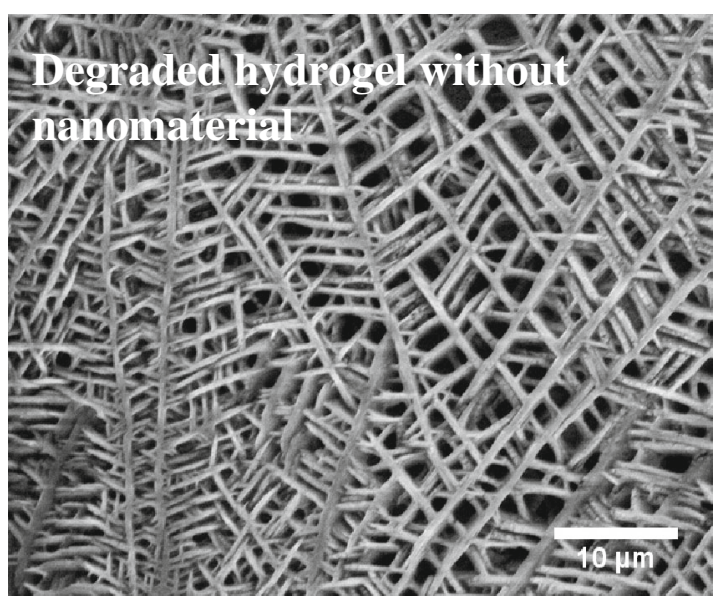

(a) Degraded hydrogel with no nanomaterial (Jia, 2011).
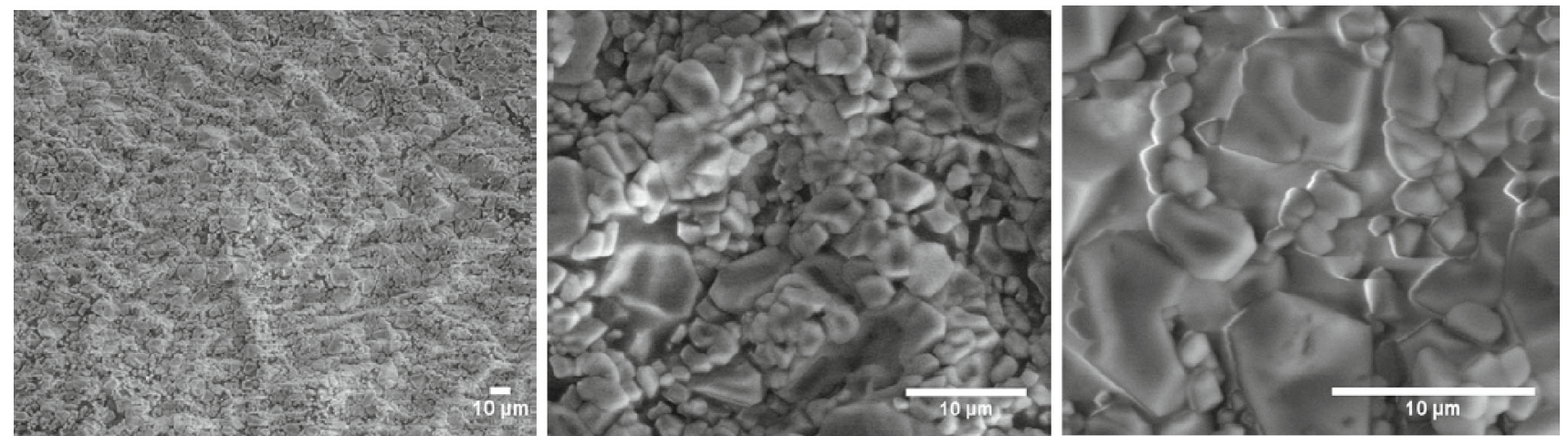

(b) Degraded 3\% $\mathrm{Ca}^{2+}$ nanocomposite PPG: tiny block-like particles are observed

Fig. 7 After-degradation environmental scanning electron microscopy (ESEM) micrographs of: a A degraded hydrogel with no nanomaterial. b A $3 \%$ degraded $\mathrm{Ca}^{2+}$ nanocomposite PPG. The initial network structure collapses into a block-like structure in degraded $\mathrm{Ca}^{2+}$ nanocomposite PPG and into ridge-like structures in degraded gel without nanomaterial. This signifies the degradation of the gel material into a polymer solution. We lack sufficient knowledge to explain this occurrence. Characterization of hydrogel network is a complex process given its 3-Dimensional complex nature and its frequent, dynamic changes to outside stimuli such as solvent, temperature, salinity, $\mathrm{pH}$ etc 




Fig. 8 Optical microscopy micrograph of degraded $0.2 \% \mathrm{Ca}^{2+}$ nanocomposite PPG. Sparse and tiny particles with an average size of 3 microns were seen throughout the sample

structure that was initially observed before degradation disappears (collapses) into block-like structures in degraded $\mathrm{Ca}^{2+}$ nanocomposite PPG and into ridge-like structures in degraded gel without nanomaterial. This signifies the degradation of the gel material into a polymer solution.

Optical microscopy imaging of $\mathrm{Ca}^{2+}$ nanocomposite PPG after degradation

After the $\mathrm{Ca}^{2+}$ nanocomposite PPG, we utilized an optical microscope to help us understand the nature of the degraded nanocomposite material. Figure 8 presents an optical micrograph of a $0.2 \% \mathrm{Ca}^{2+}$ Nanocomposite gel after degradation. Gel composition is $23 \%$ acrylamide, $100 \mathrm{ppm}$ ammonium persulfate initiator and 1,500 ppm PEG crosslinker. We observed very few and tiny particles which were sparsely scattered across the entire sample and had an average size of about 3 microns (Fig. 8). These smaller particles can travel deeper into the formation to mobilize additional oil.

\section{Conclusion}

- A nanocomposite preformed particle gel has been synthesized using Calcium Montmorillonite as nanomaterial, and evaluated for mobility control and fracture-plugging applications in mature reservoirs.

- It was observed that gel strength increased with increasing nanomaterial concentration.

- It was also observed that long-term thermal stability of hydrogels was directly proportional to nanomaterial concentration. The higher the nanomaterial concentration, the longer the thermal stability of the hydrogels.

- Calcium Montmorillonite nanocomposite hydrogel can be used for conformance control applications because they have higher strengths and long-term thermal resistance than hydrogels without nanomaterial. Thus they are potential future candidates to replace existing hydrogels for conformance control applications.

Acknowledgments Funding for this project is provided by RPSEA through the "Ultra-Deepwater and Unconventional Natural Gas and Other Petroleum Resources" program authorized by the US Energy Policy Act of 2005. RPSEA (www.rpsea.org) is a nonprofit corporation whose mission is to provide a stewardship role in ensuring the focused research, development and deployment of safe and environmentally responsible technology that can effectively deliver hydrocarbons from domestic resources to the citizens of the United States. RPSEA, operating as a consortium of premier US energy research universities, industry, and independent research organizations, manages the program under a contract with the US Department of Energy's National Energy Technology Laboratory.

Open Access This article is distributed under the terms of the Creative Commons Attribution License which permits any use, distribution, and reproduction in any medium, provided the original author(s) and the source are credited.

\section{References}

Asghari K (1999) Evaluating gel systems for permeability modification purposes in carbon dioxide flooding processes and investigating the fluid flow through hydrogels. PhD Dissertation, University of Kansas, Lawrence, KS

Bai B, Li Y, Liu X (1999) New development of water shutoff and profile control in oilfields in China. Oil Drill Product Technol 20:3

Bai B, Li L, Liu Y, Liu H, Wang Z, You C (2007a) Preformed particle gel for conformance control: factors affecting its properties and applications. SPE Reservoir Eval Eng 10(4):415-422

Bai B, Liu Y, Coste JP, Li L (2007b) Preformed particle gel for conformance control: transport mechanism through porous media. SPE Reserv Eval Eval 10:176

Bai B, Huang F, Liu Y, Seright RS, Wang Y, (2008) Case study on preformed particle gel for in-depth fluid diversion. Paper SPE 113997 presented at the SPE/DOE Symposium on Improved Oil Recovery, Tulsa, Apr 19-23

Bai B, Wei M, Liu Y (2013) Field and lab experience with a successful preformed particle gel conformance control technology. Paper SPE 164511 presented at the SPE Production and Operations Symposium, Oklahoma City, Oklahoma

Bryant SL, Rabaioli MR, Lockhart TP (1996) Influence of syneresis on permeability reduction by polymer gels. SPE 35446-PA. SPE Prod Facil 11(4):209-215

Frampton H, Morgan J, Cheung S, Munson L, Chang K, Williams D (2004) Development of a novel waterflood conformance control system. Paper SPE 89391 presented at the SPE/DOE 14th Symposium on IOR, Tulsa, OK, USA

Kytai TN, Jennifer LW (2002) Photopolymerizable hydrogels for tissue engineering applications. Biomaterials 23:4307-4314

Liu B, Bai B, Li Y (1999) Research on preformed gel grains for water shutoff and profile control. Oil drill Product Technol 21:3

Liu Y, Zhu M, Liu X, Zhang W, Sun B, Chen Y, Hans-Juergen PA (2006) High clay content nanocomposite hydrogels with surprising mechanical strength and interesting deswelling kinetics. Polymer 47:1-5 
Nelea M, Hoemann CD, Shive MS, Chenite A, Buschmann MD (2007) Ultrastructure of chitosan-glycerol phosphate hydrogels by ESEM. Microsc Microanal 13(Suppl). doi: 10.1017/ S1431927607073357

Seright RS (1990) Impact of dispersion on gel placement for profile control. Paper SPE 20127 presented at the 1990 SPE Permian Basin Oil and Gas Recovery Conference, Midland, Mar 8-9

Tongwa P, Nygaard R, Bai B (2013a) Evaluation of a nanocomposite hydrogel for water shut-off in enhanced oil recovery applications: design, synthesis, and characterization. J Appl Polym Sci 128:787-794

Tongwa P, Nygaard R, Aaron B, Bai B (2013b) Evaluation of potential fracture-sealing materials for remediating $\mathrm{CO}_{2}$ leakage pathways during $\mathrm{CO}_{2}$ sequestration. Int $\mathrm{J}$ Greenh Gas Control, JGGC-D-13-00044R1

Vossoughi S (2000) Profile modification using in-situ gelation technology-a review. J Petrol Sci Eng 26:199-209

Wang HG, Guo WK, Jiang HF (2001) Study and application of weak gel system prepared by complex polymer used for depth profile modification. Paper SPE 65379 presented at the SPE International Symposium on Oilfield Chemistry, Houston, 13-16 Feb. doi: 10.2118/65379-MS
Wang W, Liu Y, Gu Y (2003) Application of a Novel polymer system in chemical enhanced oil recovery (EOR). Colloid Polym Sci 281(11):1046-1054

Wen-Fu L, Sung-Chuan L (2006) Effect of hydrotalcite on the swelling and mechanical behaviors for the hybrid nanocomposite hydrogels based on gelatin and hydrotalcite. J Appl Polym Sci 100:500-507

Willhite GP, Green DW, Young TS, Thiele JL, Michnick MJ, Vossoughi S, Terry RE (1986) Evaluation of methods of reducing permeability in porous rocks by in situ polymer treatment-final report. DOErBCr10354-16 _DE86000-264., NTIS, US Department of Commerce, Springfield, VA, February

Young TS, Willhite GP, Green DW (1988) Study of intra molecular crosslinking of polyacrylamide in $\mathrm{Cr}(\mathrm{III})$-polyacrylamide gelation by size-exclusion chromatography, Low-Angle Laser Light Scattering, and Viscometry. In: Stahl GA, Schulz DN (eds) Water-soluble polymers for petroleum recovery. Plenum Press, New York City, pp 329-342

Zhang H, Bai B (2011) Preformed-particle-gel transport through open fractures and its effect on water flow. SPE J 16(2):388-400 